\title{
Antenna Design and Comparison of Micro ICCLMA with Circular and Rectangular Patch
}

\author{
S.Rajeswari ${ }^{1}$, C.Rekha ${ }^{2}$ \\ ${ }^{I}$ (Electronics and Communication Engineering, PET Engineering College, India \\ ${ }^{2}$ (Electronics and Communication Engineering, PET Engineering College, India)
}

\begin{abstract}
In this paper, a high gain small size antennas are micro ICCLMA (Inductively Coupled Capacitively Loaded monopole antenna) with circular patch and micro ICCLMA with rectangular patch is presented. These different types of patch antenna topologies were designed with same dimensions to produce higher gain. We begin with the comparison between the antenna designs, requirements and continue with a discussion issues and simulation results. Each antenna topology is then discussed including the limitations and advantages of each. In fact, each technique is uniquely designed to produce size reduction and higher gain antennas. Among two antennas the micro ICCLMA with circular patch provides higher gain as compared to other micro ICCLMA with rectangular patch. The antenna design simulation results were done by using CST Microwave Studio. The gain of micro ICCLMA with circular patch is $46.03 \mathrm{~dB}$.
\end{abstract}

Keywords - Antenna radiation patterns, lumped elements, monopole antennas, patch antennas, vertically polarized antennas.

\section{INTRODUCTION}

In modern research on high gain small size antennas has been developed by an increasing interest in the use of wireless communication applications. For near ground communications where both the transmit and receive antennas are placed near the ground is by many orders of magnitude lower than any other antenna orientation configurations [12]. Applications such as unattended ground sensors (UGS), vertically polarized antennas with omnidirectional radiation pattern are highly desired. As the antenna size reduction is obviously major problem in wireless communication devices. Therefore several methods have been investigated for extremely short monopole antennas with very high lateral dimensions, while maintaining high radiation efficiency. With the development of wireless communication devices and mobile phone technology, it has become significant to provide low profile antennas with omnidirectional radiation pattern [6]. There are various size reduction techniques used in the design of small antennas in which reactive inductive loading and reactive capacitive loading. In [7], electrically small antennas are further investigated and the performance such as impedance matching, the radiation pattern, the radiation efficiency, quality factor $(\mathrm{Q})$, and polarization to be reported. In [8], the antenna is characterized with fractal geometries and the performance, it can be summarized that increasing the fractal dimension of the antenna leads to a higher degree of miniaturization. Applications of fractal geometry are becoming mostly used in the fields of science and engineering. Antenna miniaturization can generally be categorized into two methods are Miniaturizing the antenna topology using space filling compression technique and Antenna miniaturization using magneto-dielectric materials [9]. The spatial network Method [18]-[19] provides strong radiation with an omnidirectional pattern in the horizontal direction. The dielectric truncation [14]-[15] is not close to the source, and then the space wave power is unaffected. It gives better efficiency. For certain applications, where the bandwidth can be compromised, it is found that by a comprehensive analysis of a new wide bandwidth compact antenna called (WC) wide compact J-pole antenna provided 50\% impedance bandwidth [10]. Although these exist many antenna miniaturization techniques, most of them cannot provide high gain. However it is difficult to implement in practice, because these antennas include a multilayer geometry. An extremely (LMMMA) low profile multi element miniaturized monopole antenna [20] based on superposition of multiple quarter-wave segments that are meandered and spiraled around to suppress the radiation from horizontal currents above the ground plane. The LMMMA produces purely vertically polarization which leads to lower gain. Recently, a low profile antenna called micro inductively coupled capacitively loaded monopole antenna (ICCLMA) in which the techniques such as in-plane capacitive coupling, top loading, shorting pin achieve improved polarization purity and high gain with antenna miniaturization. The micro ICCLMA with circular patch and micro ICCLMA with rectangular patch were compared and the simulation results were obtained.

\section{OVERVIEW OF ANTENNAS}

Miniaturized antennas offer several advantages over classical antennas like low cost, easy to design, planar shape, conformability, etc. First we investigate unidirectional cylindrical monopole antenna [21] with 
$40 \mathrm{~mm}$ diameter and $25 \mathrm{~mm}$ height. Here the radiation pattern is unidirectional, in which the major radiation is in the backward direction, off the apex of the antenna. The overall frequency range of this antenna is between $500 \mathrm{MHz}$ and $2 \mathrm{GHz}$. The cylindrical conformal monopole antenna includes the lumped elements are loaded $\mathrm{R}$ (resistance) and C (capacitor). These parameters are used to minimize reflections of the antenna. The lumped elements are serially connected. The value of resister and capacitor is $100 \Omega$ and $1.5 \mathrm{pF}$. By using this lumped element approach impedance transformation can be accomplished easily.

Although there are many antenna approaches, most of them have lower gain and poor polarization purity. The new antenna topology called Micro ICCLMA. Micro ICCLMA is the very small size antenna of dimension $3.393 \mathrm{~mm} \times 0.0474 \mathrm{~mm}$. It includes the lumped elements values are related to the diameter of two pins and the width, length and height of metal sheets, respectively. The perfect electric conductor (PEC) material is used for simulation. Micro ICCLMA is used typically at frequencies between 2 to $3 \mathrm{GHz}$. The dimensions of the antenna are chosen based on the system size requirements.

\section{III.}

ANTENNA DESIGN

\subsection{Micro ICCLMA with rectangular patch}

The proposed micro ICCLMA with three layers of total dimension is $3.393 \times 0.0474 \mathrm{~mm}$ using CST. The vertical feeding pin and shorting pin were inserted in the middle and top layer respectively. The diameters of the pins were $0.5 \mathrm{~mm}$. In newly developed ICCLMA, the same equivalent circuit model is to be followed by changing the lumped elements values corresponding to their enlarged gain value.

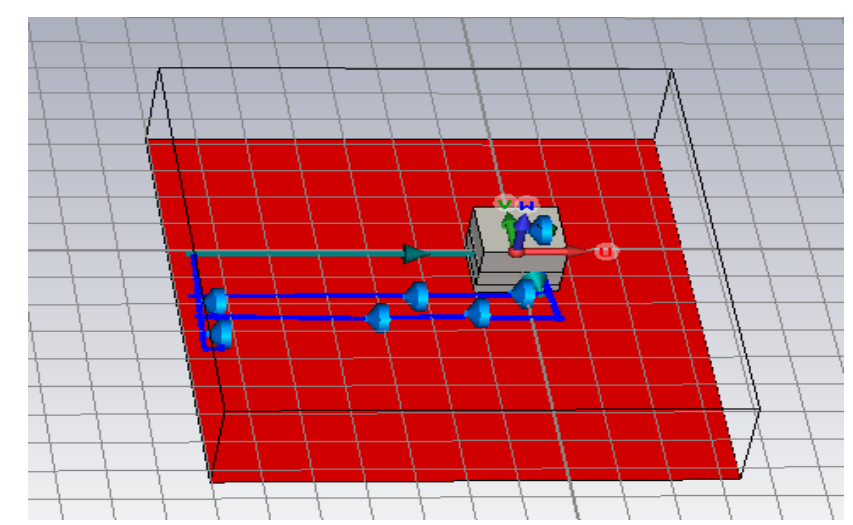

Fig. 1. Micro ICCLMA design with lumped elements

The inductors $\mathrm{L} 1=5 \mathrm{nH}, \mathrm{L} 2=5 \mathrm{nH}$ were placed in the feeding pin and shorting pin respectively and the additional inductors are $\mathrm{L} 3=1.9 \mathrm{nH}, \mathrm{L} 4=1 \mathrm{nH}$ were inserted in the top and middle layers respectively. The shunt capacitor $\mathrm{C} 1=0.788 \mathrm{pF}$ was placed in the metallic patch. Here the additional capacitors $\mathrm{C} 2=1 \mathrm{pF}, \mathrm{C} 3=1 \mathrm{pF}$ were inserted into the layers because the metallic trace is narrow. The resistor $\mathrm{R}=1.5 \Omega$ was inserted into the top layer. The antenna dimensions (length and width) were calculated by using the formula as given by,

$$
\begin{aligned}
& \mathbb{L}=\frac{\mathbb{L}_{0}}{2}=2 \Delta \mathbb{L} \\
& \mathrm{L}=3.393 \mathrm{~mm}
\end{aligned}
$$

where $\Delta L$ denotes the dimension of the patch and $L$ denotes the actual length of the antenna.

where $\mathrm{h}$ represents the height of the substrate.

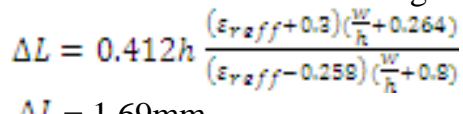

$$
\begin{aligned}
& \Delta L=1.69 \mathrm{~mm}
\end{aligned}
$$

Width of the antenna is calculated by using the formula is given by,

$$
\begin{aligned}
& W=\frac{c}{2 f_{b}}\left[\sqrt{\frac{2}{\left(\varepsilon_{\mathrm{r}}+1\right)}}\right] \\
& W=0.0474 \mathrm{~mm}
\end{aligned}
$$

where $\varepsilon_{\mathbb{F}}$ represents the dielectric constant, f represents the frequency range, and $C$ represents the velocity of light.

The effective dielectric constant ( $\left.\varepsilon_{\text {waff }}\right)$ is given by,

$$
\begin{aligned}
& \varepsilon_{\text {veff }}=\frac{\varepsilon_{\mathrm{Y}}+1}{2}+\frac{\varepsilon_{\mathrm{p}}-1}{2}\left[1+12 \frac{\mathrm{i}}{\mathrm{W}}\right]^{1 / 2 / 2} \\
& \varepsilon_{\text {yeff }}=2.21
\end{aligned}
$$




\subsection{Micro ICCLMA with Circular Patch}

The proposed micro ICCLMA with circular patch of total dimension is $3.393 \times 0.0474 \mathrm{~mm}$ using CST. The design procedure for micro ICCLMA as well as the micro ICCLMA with circular patch is similar. But the main difference between these two types of antenna is patch. In this type of antenna using the patch as circular. Here the circular patch radius is $1.6765 \mathrm{~mm}$ and the height is $1 \mathrm{~mm}$. The lumped elements were connected corresponding to the equivalent circuit model of ICCLMA antenna.

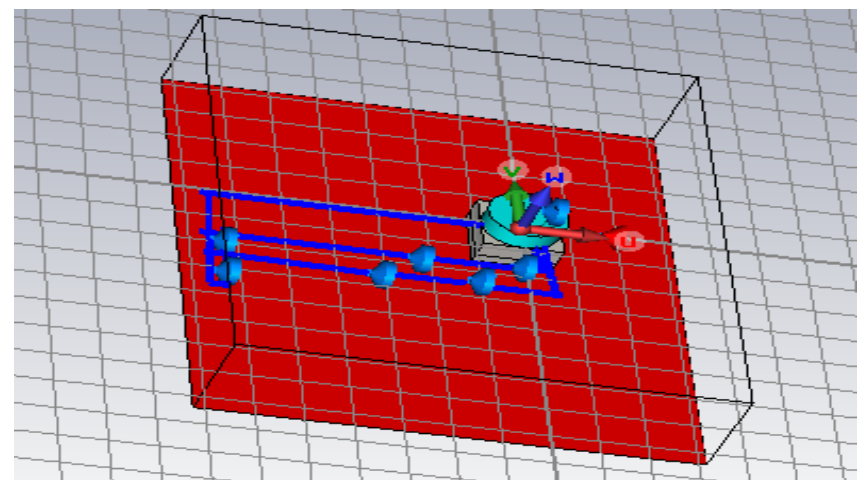

Fig. 2. Micro ICCLMA with circular patch design

Antenna input impedance and admittance of the antenna were obtained is given by,

$$
\begin{gathered}
X_{f}=\frac{\pi}{2 \sqrt{\varepsilon_{\text {gaff }}}} \\
Z_{f}=1.14 \mathrm{~m} \Omega \\
Y_{f}=w / 2 \\
Y_{f}=0.237 \mathrm{mho}
\end{gathered}
$$

where $\mathrm{Xf}$ and $\mathrm{Yf}$ is the antenna input impedance and admittance.

\section{SimUlation RESULTS}

I ) We designed the micro ICCLMA with rectangular patch using CST software and the corresponding simulation results were shown in below figure.

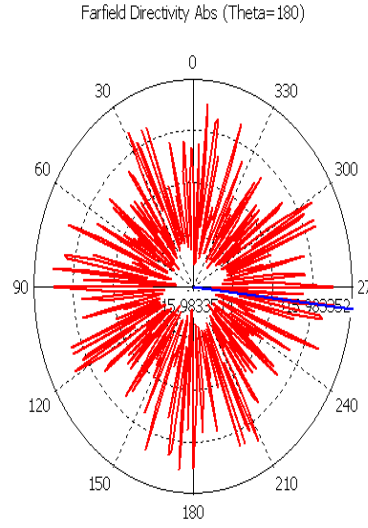

Phi / Degree vs. dBi

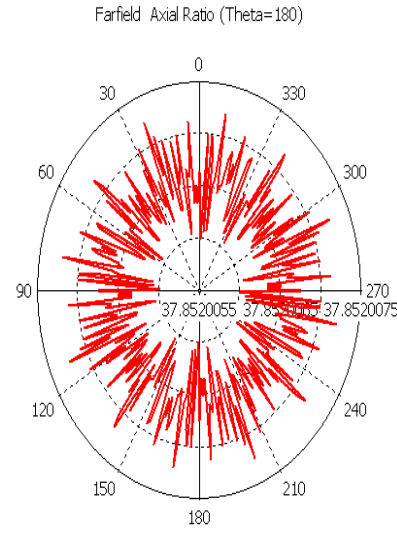

Phi / Degree vs, dB

(b)

Fig. 3. Simulated (a) Farfield directivity abs $\left(\theta=180^{\circ}\right)$ and (b) Farfield axial ratio $\left(\theta=180^{\circ}\right)$ in micro ICCLMA with rectangular patch design.

In the above figure 3 (a) shows farfield directivity absolute value at $\theta=180^{\circ}$. It provides the omnidirectional radiation pattern. Here the frequency as $2.5 \mathrm{GHz}$ and the main lobe magnitude value as $316.0 \mathrm{~dB}$. Fig. 3(b) shows the farfield directivity axial ratio at $\theta=180^{\circ}$. The main lobe magnitude as $37.9 \mathrm{~dB}$. 


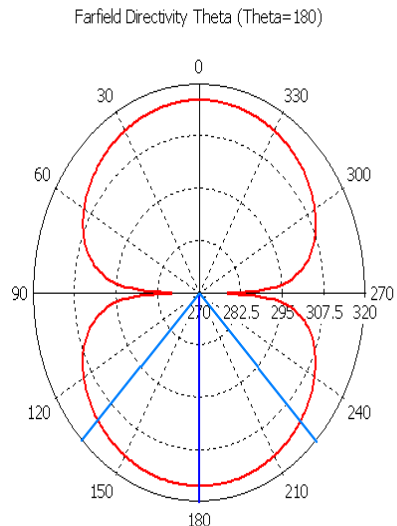

Phi / Degree vs, dBi

(a)

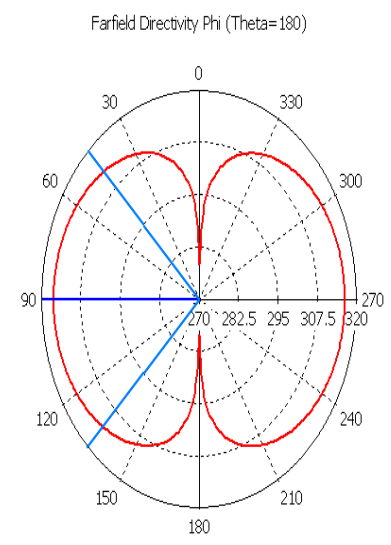

Phi / Degree vs. dBi

(b)

Fig. 4. Simulated (a) Farfield directivity theta $\left(\theta=180^{\circ}\right)$ and (b) Farfield directivity phi $\left(\theta=180^{\circ}\right)$ in micro ICCLMA with rectangular patch design.

The corresponding farfield directivity theta at $\theta=180^{\circ}$ is shown by Fig. 4(a). It provides the figure of eight radiation pattern and the main lobe magnitude as $316.0 \mathrm{~dB}$, the main lobe direction as $180.0 \mathrm{deg}$ and the angular width as $89.9 \mathrm{deg}$. Fig. 4(b) shows the Farfield directivity phi $\theta=180^{\circ}$. Here the main lobe magnitude as $316.0 \mathrm{~dB}$, the main lobe direction as $90.0 \mathrm{deg}$ and the angular width as $89.9 \mathrm{deg}$. The gain of this micro ICCLMA is $45.2 \mathrm{~dB}$.

II ) We designed the micro ICCLMA with circular patch topology using CST software and the corresponding simulation results were shown in below figure.

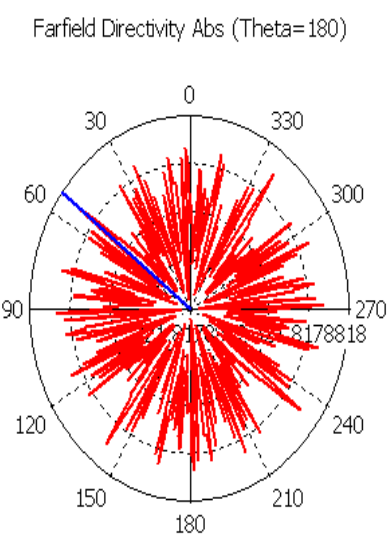

Phi / Degree vs. dBi

(a)
Farfield Axial Rato (Theta=180)

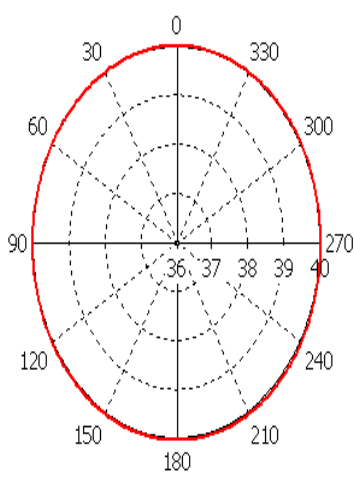

Phi / Degree vs. dB

(b)

Fig. 5. Simulated (a) Farfield directivity abs $\left(\theta=180^{\circ}\right)$ and (b) Farfield axial ratio $\left(\theta=180^{\circ}\right)$ in micro ICCLMA with circular patch design.

In the above figure 5(a) shows farfield directivity absolute value at $\theta=180^{\circ}$. It provides the omnidirectional radiation pattern. Here the frequency as $2.5 \mathrm{GHz}$ and the main lobe magnitude value as $321.8 \mathrm{~dB}$. Fig. 5(b) shows the farfield directivity axial ratio at $\theta=180^{\circ}$. The main lobe magnitude as $40 \mathrm{~dB}$. 


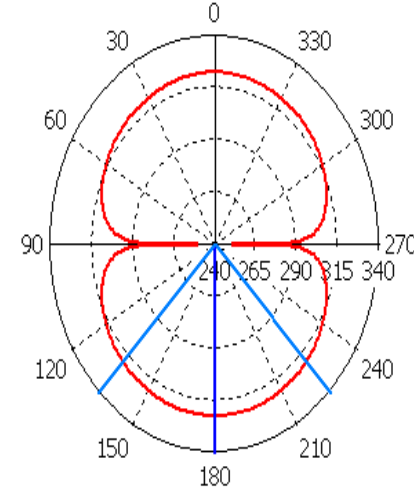

Phi / Degree vs, dBi

(a)

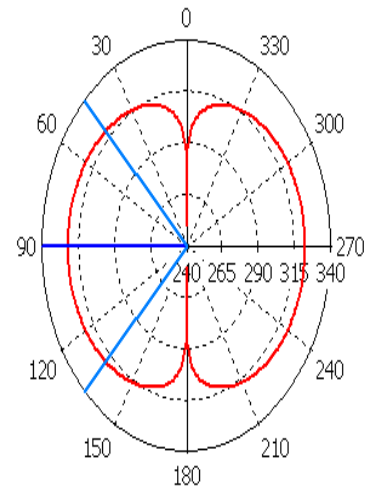

Phi / Degree vs. dBi

(b)

Fig. 6. Simulated (a) Farfield directivity theta $\left(\theta=180^{\circ}\right)$ and (b) Farfield directivity phi $\left(\theta=180^{\circ}\right)$ in micro ICCLMA with circular patch design.

The corresponding farfield directivity theta at $\theta=180^{\circ}$ is shown by Fig. 6(a). It provides the figure of eight radiation pattern and the main lobe magnitude as $321.8 \mathrm{~dB}$, the main lobe direction as $180.0 \mathrm{deg}$ and the angular width as $89.9 \mathrm{deg}$. Fig. 6(b) shows the Farfield directivity phi $\theta=180^{\circ}$. Here the main lobe magnitude as $321.8 \mathrm{~dB}$, the main lobe direction as $90.0 \mathrm{deg}$ and the angular width as $89.9 \mathrm{deg}$. The gain of this micro ICCLMA with circular patch is $46.03 \mathrm{~dB}$. The proposed micro ICCLMA with circular patch provides higher gain as compared to the existing ICCLMA antennas.

V. TABLE

Comparison between micro ICCLMA with rectangular patch and micro ICCLMA circular patch design Output Parameters

\begin{tabular}{|c|c|c|}
\hline Parameters & $\begin{array}{c}\text { MicroICCLMA with Rectangular } \\
\text { Patch }\end{array}$ & MicroICCLMA with Circular Patch \\
\hline Dimension & $3.393 \times 0.0474 \mathrm{~mm}$ & $3.393 \times 0.0474 \mathrm{~mm}$ \\
\hline $\begin{array}{c}\text { Main lobe } \\
\text { magnitude }\end{array}$ & $316.0 \mathrm{dBi}$ & $321.8 \mathrm{dBi}$ \\
\hline Gain & $45.2 \mathrm{~dB}$ & $46.03 \mathrm{~dB}$ \\
\hline Beam width & $89.9^{\circ}$ & $89.9^{\circ}$ \\
\hline $\begin{array}{c}\text { Main lobe } \\
\text { direction }\end{array}$ & $90.0^{\circ}$ & $90.0^{\circ}$ \\
\hline $\begin{array}{c}\text { Frequency } \\
\begin{array}{c}\text { S-Parameter } \\
\text { magnitude in } \\
\text { dB }\end{array}\end{array}$ & $2.5 \mathrm{GHz}$ & $2.5 \mathrm{GHz}$ \\
\hline
\end{tabular}

\section{CONCLUSION}

In this paper, antenna design and parameters comparison of micro ICCLMA with rectangular patch and micro ICCLMA with circular patch were presented using CST and the radiation pattern performance of the Eplane and H-plane pattern are obtained. The micro ICCLMA with rectangular patch and micro ICCLMA with circular patch topology provides better reduction in size, improved polarization purity and high gain compared to other existing antennas. The reduced size of the micro ICCLMA with rectangular patch and circular patch antenna is suitable for mobile radio communications, wireless communications.

\section{Acknowledgements}

I have taken efforts in this paper. However, it would not have been possible without the kind support and help of many individuals. I would like to extend my sincere thanks to all of them. I am highly indebted to Mrs.C.Rekha for her guidance and constant supervision as well as for providing necessary information regarding the project and also for her support in completing the paper. I owe a sincere prayer to the LORD 
ALMIGHTY for his kind blessings and giving me full support to do this work, without which would have not been possible. My thanks and appreciations also go to my colleague in developing the paper and people who have willingly helped me out with their abilities.

\section{REFERENCES}

[1] Jungsuek Oh and Kamal SarabandI (2012)," Low Profile, Miniaturized Antenna", IEEE Trans. Antennas Propag., vol. 57, no.1,pp. 1206-1213.

Inductively Coupled Capacitively Loaded Monopole

[2] Hong W, Behdad N, and Sarabandi K (2004) "Size reduction of cavity backed slot antennas", IEEE Trans. Antennas Propag., vol. 54, pp. 1461-1466.

[3] Behdad N and Sarabandi K (2004), "Bandwidth enhancement and further size reduction of a class of miniaturized slot antennas", IEEE Trans. Antennas Propag., vol. 52, pp. 1928-1935.

[4] Goubau G (1976), "Multielement monopole antennas", in Proc. Workshop on Electrically Small Antennas ECOM, Ft., Monmouth, NJ, pp.63-67.

[5] Rowell C.R and Murch R. D (1997), “A capacitively loaded PIFA for compact mobile telephone handsets”, IEEE Trans. Antennas Propag., vol.45, pp. 837-842.

[6] Hala Elsadek (2011), "Microstrip Antennas for Mobile Wireless Communication Systems" CC BY-NC-SA 3.0 license, in subject Electrical and Electronic Engineering.

[7] Steven R.Best (2011), "A Discussion on Electrically Small Antennas Loaded with High Permittivity and Permeability Materials", The MITRE Corporation.

[8] Kulbir Singh, Vinit Grewal, Rajiv Saxena_(2009), "Fractal Antennas: A Novel Miniaturization Technique for Wireless Communications", ECED, Thapar University, Patiala, India; National Institute of Technology, Jalandhar, India; Jay Pee Institute of Engineering and Technology, Raghogarh, Guna, M.P, India.

[9] Brad A. Kramer (2007), "Size reduction of an UWB low-profile spiral antenna", Ohio State University, Electrical Engineering.

[10] Minh-Chau Huynh (2004), "Wideband compact antennas for wireless communication applications", Virginia Polytechnic Institute and State University,

[11] Chiu C.Y, Shum K.M and Chan C.H (2007), "A tunable via-patch loaded PIFA with size reduction", IEEE Trans. Antennas Propag., vol. 55, no.1, pp. 65-71.

[12] Liao D and Sarabandi K (2008), “Terminal-to-terminal hybrid full-wave simulation of low-profile, electrically-small, near-ground antennas", IEEE Trans. Antennas Propag., vol. 56, no. 3, pp. 806-814.

[13] Liao D and Sarabandi K (2005) "Optimization of low-profile antennas for applications in unattended ground sensor networks", IEEE Trans. Antennas Propag., vol. 53, no. 11, pp. 3747-3756.

[14] Bhattacharyya (1991), "Effects of ground plane and dielectric truncations on the efficiency of a printed structure", IEEE Trans. Antennas Propag. vol. 39, pp. 303-308.

[15] Huynh M.C. and Stutzman W (2003), "Ground plane effects on the planar inverted-F antenna (PIFA) performance", IEE Proc. Microwave Antennas Propag., vol. 150, no. 4, pp. 209-213.

[16] Herscovivi N and Diadem E (1999), "Omni directional antennas for wireless communication”, in Proc. IEEE Int. Symp. Antennas Propag. vol. 1, pp. 556-559.

[17] McLean J, Foltz H, and Crook G(1999), "Broadband, robust, low profile monopole incorporating top loading, dielectric loading, and a distributed capacitive feed mechanism”, in Proc. IEEE Int. Symp. Antennas Propag, vol. 3, pp. 1562-1565.

[18] Taga T and Tsunoda K (1991), “Analysis of a planar inverted-F antenna by spatial network method”, IEICE, B-2, vol. J74-B-2, no. 10 , pp.538-545.

[19] Sekine S, Ito T, Odachi N, Murakami Y and Shoki H (2003), "Design method for a broadband inverted-F antenna by parallel resonance mode", IEICE, B, vol. J86-B, no. 9, pp. 1806-1815.

[20] Hong W.B and Sarabandi K (2009), "Low-profile, multi-element, miniaturized monopole antenna”, IEEE Trans. Antennas Propag., vol. 57, no.1, pp. 72-80.

[21] Yan-Tao Li, Xiao-Lin Yang, Zhao-Bo Li, Lei Wang, and Hong-Chun Yang (2011).” A unidirectional cylindrical conformal monopole antenna designed for impulse radar system”, IEEE Antennas and wireless propagation letters. Vol. 10, pp. 1397-1400. 